Pacific Journal of Mathematics

APPROXIMATIONS TO REAL ALGEBRAIC NUMBERS BY
ALGEBRAIC NUMBERS OF SMALLER DEGREE 


\title{
APPROXIMATIONS TO REAL ALGEBRAIC NUMBERS BY ALGEBRAIC NUMBERS OF SMALLER DEGREE
}

\author{
JOHN F. MORRISON
}

If $\beta$ is an algebraic number of degree $n+1$ then the number of solutions $\alpha$, with $\alpha$ algebraic of degree at most $n$, to the inequalities

$$
|\beta-\alpha|<\varphi(H(\alpha)), \quad 1 \leqq H(\alpha) \leqq B
$$

is studied using methods developed by Schmidt and Adams for counting solutions to inequalities involving linear forms. In (1) $H(\alpha)$ is a height function which differes slightly from the usual height and $\varphi$ is a function which decreases to zero. If $\varphi(y) y^{n+1} \rightarrow \infty$ as $y \rightarrow \infty$ then the number of solutions is given as an integral plus an error term. If $\varphi(y) y^{n+1}$ is constant then the number of solutions is either bounded or asymptotic to $C \log B$ for some constant $C$.

1. Introduction and statement of theorems. In this paper the methods of Schmidt [5] and Adams [1, 2], for counting solutions to inequalities involving linear forms with algebraic coefficients will be adapted to prove two theorems on the approximation of algebraic numbers by algebraic numbers of smaller degree.

In what follows $\beta$ will denote a fixed real algebraic number of degree $n+1, \varphi(y)$ a decreasing function which is positive for $y>0$ and which tends to zero, and $P(T)$ a polynomial of degree $k \leqq n$ with integer coefficients. If

$$
P(T)=q_{k} T^{k}+\cdots+q_{1} T+q_{0}
$$

then the height of $P$ is defined by $H(P)=\max \left(\left|q_{1}\right|, \cdots,\left|q_{k}\right|\right)$. This is not the usual definition of height, but it is more convenient for technical reasons. In the situation we are considering we will have $q_{0}$ uniquely determined by $q_{1}, \cdots, q_{k}$ and $\left|q_{0}\right|=O(H(P))$. If $\alpha$ is an algebraic number whose minimal polynomial over $Z$ is $P(T)$ then the height of $\alpha$ is defined by $H(\alpha)=H(P)$. The number of solutions to

$$
|\beta-\alpha|<\varphi(H(\alpha)), \quad 1 \leqq H(\alpha) \leqq B
$$

with $\alpha$ algebraic of degree at most $n$ will be denoted by $\Gamma(\varphi, B)$. There are two cases in which we will find $\Gamma(\varphi, \beta)$.

THEOREM 1. Assume that, as $y \rightarrow \infty, \chi(y)=\varphi(y) y^{n+1} \rightarrow \infty$ and $\varphi(y) y^{n+\delta} \rightarrow 0$ for some $\delta>0$. If $n \geqq 2$ we have 


$$
\begin{aligned}
\Gamma(\varphi, B)= & C \int_{\max \left|y_{2}\right| \leqq B} \varphi\left(\max \left|y_{i}\right|\right) \\
& \times\left|n y_{n} \beta^{n-1}+(n-1) y_{n-1} \beta^{n-2}+\cdots+y_{1}\right| d y_{1} \cdots d y_{n} \\
& +O\left(\int_{1}^{B} y^{n} \varphi(y) \chi(y)^{-1 /(n+1)} d y\right) .
\end{aligned}
$$

In the case $\chi(y)$ is constant we get:

Theorem 2. There exists a constant $C_{0}$ such that if $C>C_{0}$ then there is a $C^{\prime}>0$ with $\Gamma\left(C y^{-(n+1)}, B\right) \sim C^{\prime} \log B$ and if $C<C_{0}$ then $\Gamma\left(C y^{-(n+1)}, B\right)=O(1)$.

2. A related problem. What we will actually count is $\Delta(\varphi, B)$, which is the number of primitive polynomials of degree at most $n$ such that

$$
0<P(\beta)<\left|P^{\prime}(\beta)\right| \varphi(H(P)), \quad 1 \leqq H(P) \leqq B
$$

wxere $P^{\prime}(T)$ is the derivative of $P(T)$. In order to get the theorems of the previous section we now prove:

THEOREM 3. If $\chi(y)$ is bounded below and $\varphi(y) y^{n+\dot{o}} \rightarrow 0$ for some $\delta>0$ then

$$
\Delta\left(\left(1-C y^{-(1+\hat{o})}\right) \varphi, B\right) \leqq \Gamma(\varphi, B) \leqq \Delta\left(\left(1+C y^{-(1+\hat{o})}\right) \varphi, B\right)
$$

where the constant $C$ depends on $\beta$ and $\delta$.

Before beginning the proof we set up some notation. If $P$ is any polynomial with $P(\alpha)=0$ then we let $\alpha=\alpha_{1}$ and denote the other roots of $P$ by $\alpha_{2}, \cdots, \alpha_{k}$. We use $f \ll g$ to indicate $f<C g$ where $C$ may depend on $\beta$ and $\delta$. $C$ will denote a constant which will not always be the same each time it appears.

We will use the following facts:

$$
\left|\alpha-\alpha_{i}\right|-|\beta-\alpha| \leqq\left|\beta-\alpha_{i}\right| \leqq\left|\alpha-\alpha_{i}\right|+|\beta-\alpha| \text {. }
$$

If $P(\alpha)=0$ then (1) is equivalent to

$$
|P(\beta)| \leqq\left|q_{k}\left(\beta-\alpha_{2}\right) \cdots\left(\beta-\alpha_{k}\right)\right| \varphi(H(\alpha)) .
$$

(5) Since $\rho$ is bounded above there are only a finite number of $\alpha$ satisfying (1) with $H(\alpha)$ less than a given bound.

We now prove some lemmas.

LEMMA 1. If $P(\alpha)=0$ then $|\alpha| \gg H(P)^{-1}$. 
Proof. If $|\alpha| \geqq 1$ this is clear. If not, since $q_{k} \alpha^{k}+\cdots+q_{0}=0$ we get

$$
\left|q_{0} \alpha^{-1}\right|=\left|q_{k} \alpha^{k-1}+\cdots+q_{1}\right| \ll H(P)
$$

which proves the lemma.

Lemma 2. If $P(\alpha)=0$ and $|\beta-\alpha|$ is bounded then

$$
\left|\alpha_{i}\right| \ll H(P), \quad 1 \leqq i \leqq k .
$$

Proof. If $\left|\alpha_{i}\right| \leqq 1$ the result is clear. If not, since $P(\alpha)=0$ and $|\beta-\alpha|$ is bounded, then $\left|q_{0}\right| \ll H(P)$ and so $\left|q_{k} \alpha_{i}\right|=\mid q_{k-1}+\cdots+$ $q_{0} \alpha^{-(k+1)} \mid \ll H(P)$ as desired.

Lemma 3. If $|\beta-\alpha|$ is bounded and $\alpha, \alpha_{i}, \alpha_{j}$ are roots of $P$ with $\alpha_{i} \neq \alpha_{j}$ then

$$
\left|\alpha_{i}-\alpha_{j}\right| \gg H(P)^{-(k-1)}
$$

Proof. Since the proof is similar to Lemma 1 of [4] we only sketch it. The polynomial

$$
Q(T)=q_{i}^{2 k-2} \prod_{i<j}\left\{T-\left(\alpha_{i}-\alpha_{j}\right)^{2}\right\}
$$

has integer coefficients and roots $\left(\alpha_{i}-\alpha_{j}\right)^{2}$, so by Lemma 1 we have $\left|\alpha_{i}-\alpha_{j}\right|^{2} \gg H(Q)^{-1}$. By examining the coefficients of $Q$ we see $H(Q) \gg H(P)^{2 k-2}$ which gives the desired result.

LEMMA 4. (1) implies

$$
0<P(\beta)<\left|P^{\prime}(\beta)\right| \varphi(h(P))\left(1+C H(P)^{-(1+\delta)}\right)
$$

where $P$ is the minimal polynomial for $\alpha$ over $Z$, which is chosen so that $P(\beta)>0$.

Proof. From (1), (3) and Lemma 3 we get

$$
\begin{aligned}
\left|\frac{\beta-\alpha}{\beta-\alpha_{i}}\right| & \ll \frac{\varphi(H(\alpha))}{H(\alpha)^{-(k-1)}-\varphi(H(\alpha))}=\left(\frac{H(\alpha)^{1+\delta}}{H(\alpha)^{n+\delta} \varphi(H(\alpha))}-1\right)^{-1} \\
& \ll \varphi(H(\alpha))^{-(1+\delta)}
\end{aligned}
$$

since $H(\alpha)^{n+o} \varphi(H(\alpha))$ is bounded above. Thus

$$
\begin{aligned}
\left|\frac{x_{k}(\beta-\alpha) \cdots\left(\beta-\alpha_{k}\right)}{P^{\prime}(\beta)}\right| & =\left|1+\sum_{i \neq 1} \frac{\beta-\alpha}{\beta-\alpha_{i}}\right| \leqq \frac{1}{1-C H(\alpha)^{-(1+\delta)}} \\
& \leqq 1+C H(\alpha)^{-(1+\delta)} .
\end{aligned}
$$

Since (1) is equivalent to (4) this proves the lemma. 
From the preceding lemmas we see that each solution to (1) gives rise to a solution to (2) with $\varphi(H(\alpha))$ replaced by $\left(1+C H(P)^{-(1+o)}\right) \varphi(H(P))$, if $H(P)$ is large. To show

$$
\Gamma(\varphi, B) \leqq \Delta\left(\left(1+C y^{-(1+\hat{o})}\right) \varphi, B\right)+O(1)
$$

we will show that, if $H(\alpha)$ is large, all these solutions are distinct, i.e., two conjugates cannot both satisfy (1). Assume this is not true, then $P\left(\alpha_{1}\right)=P\left(\alpha_{2}\right)=0$ where $\alpha_{1}$ and $\alpha_{2}$ satisfy (1). Thus

$$
|P(\beta)| \leqq\left|x_{k}\right| \varphi(H(P))^{2} \prod_{i>2}\left|\beta-\alpha_{i}\right|
$$

which by Lemma 2 implies

$$
|P(\beta)| \ll \varphi(H(P))^{2} H(P)^{n-1} .
$$

Since $\beta$ is algebraic of degree $n+1$ we also have

$$
|P(\beta)| \gg H(P)^{-n}
$$

because the norm of $P(\beta)$ is bounded below. Comparing these two bounds gives

$$
1 \ll \varphi(H(P)) H(P)^{n-1 / 2}
$$

which contradicts $\varphi(y) y^{n+\delta} \rightarrow 0$ and so gives the desired inequality.

For the other inequality of Theorem 3 we start with a polynomial $P$ of degree $k \leqq n$ and let $\alpha=\alpha_{1}$ be a root of $P$ nearest to $\beta$. Then (2) is equivalent to

$$
|\beta-\alpha| \leqq\left|1+\sum_{i \neq 1} \frac{\beta-\alpha}{\beta-\alpha_{i}}\right| \varphi(H(P))
$$

which implies $|\beta-\alpha|$ is bounded. Thus (3) and $\varphi(y) y^{n-\hat{o}} \rightarrow 0$ imply as above

$$
\left|1+\sum_{i \neq 1} \frac{\beta-\alpha}{\beta-\alpha_{i}}\right| \leqq 1+C H(P)^{-(1+\delta)} .
$$

Also using $|\beta-\alpha|$ bounded we can show that $H(P)$ can be assumed to be large.

LemMa 5. If $\alpha$ is as above then no other root of $P$ can satisfy $\left|\beta-\alpha_{i}\right| \ll \varphi(H(P))$ for $H(P)$ large, in particular $\alpha \neq \alpha_{i}$ for $i \neq 1$.

Proof. As above we can use $|P(\beta)|$ bounded below and Lemma 2 to get $1 \ll \varphi(H(P)) H(P)^{n-1 / 2}$ which is a contradiction of $H(P)$ is large enough.

We now have that each solution to (2) gives at least one solution to

$$
|\beta-\alpha| \leqq(1+C H(P))^{-(1+\hat{o})} \varphi(H(P)) .
$$


We will now show that we get only one solution and that $H(P)$ can be replaced by $H(\alpha)$. This follows easily from:

Lemma 6. If $H(P)$ is large then (2) implies that the degree of $\alpha$ is $n$ and so $P$ is the minimal polynomial for $\alpha$. Since $P(\beta)>0$ there is only one $P$ giving rise to $\alpha$.

Proof. Assume the degree of $\alpha$ is at most $n-1$, so by a theorem of Schmidt [6, Theorem 2], $|\beta-\alpha| \gg H(\alpha)^{-(n+o)}$. If $Q(T)$ is the minimal polynomial for $\alpha$ then $P(\alpha)=0$ implies $Q \mid P$ and so

$$
H(Q)=H(\alpha) \ll H(P) \quad[3, \text { page 14] }
$$

Thus, with (2) and Schmidt's Theorem, imply

$$
H(\alpha)^{-(n+\tilde{o})} \ll \varphi(H(P)) \ll \varphi(C H(\alpha))
$$

which contradicts $\varphi(y) y^{n+\delta} \rightarrow 0$. Finally we note that, since $P$ is primitive and $P(\beta)>0, P$ is unique.

To get the desired inequality we let

$$
\varphi^{\prime}(y)=\left(1+C y^{-(1+i)}\right) \varphi(y) .
$$

What we have shown above is

$$
\Delta(\varphi, B) \leqq \Gamma\left(\left(1+C y^{-(1+\hat{)})}\right) \varphi, B\right)+O(1) .
$$

This implies

$$
\Delta\left(\left(1-C y^{-(1+\delta)}\right) \varphi^{\prime}, B\right) \leqq \Gamma\left(\varphi^{\prime}, B\right)+O(1)
$$

which finishes the proof of Theorem 3 .

3. The proof of Theorem 1. Instead of Theorem 1 we prove the following, from which Theorem 1 easily follows.

THEOREM 1'. Let $1, \beta_{1}, \cdots, \beta_{n}$ be a basis of a real number field $K$ of degree $n+1$. Assume $\varphi(y)$ is decreasing and $\chi(y)=\varphi(y) y^{n+1}$ tends to infinity. Let $L\left(y_{1}, \cdots, y_{n}\right)$ be a linear form and let $N(B)$ be the number of solutions to

$$
\begin{aligned}
0 & <q_{1} \beta_{1}+q_{2} \beta_{2}+\cdots+q_{n} \beta_{n}-p \\
& <\varphi\left(\max q_{i}\right)\left|L\left(q_{1}, \cdots, q_{n}\right)\right|, \quad 1 \leqq q_{i} \leqq B
\end{aligned}
$$

where $p, q_{1}, \cdots, q_{n} \in Z$ are relatively prime. If $n \geqq 2$

$$
\begin{aligned}
N(B)= & C \int_{1 \leqq y_{i} \leqq B} \varphi\left(\max y_{i}\right)\left|L\left(y_{1}, \cdots, y_{n}\right)\right| d y_{1} \cdots d y_{n} \\
& +O\left(\int_{1}^{B} y^{n} \varphi(y) \chi(2 y)^{1 /(n+1)} d y\right) .
\end{aligned}
$$


The proof is similar to that of Schmidt [5] and so some of the details will be omitted. For any $\xi \in K$ we let $\xi=\xi^{(0)}$ and denote its conjugates by $\xi^{(i)}, 1 \leqq i \leqq n$. Let $r+2 s=n$ and let

$$
\xi^{*(i)}= \begin{cases}\xi^{(i)} & 0 \leqq i \leqq r \\ \operatorname{Re}\left(\xi^{(i)}\right) & r+1 \leqq i \leqq r+s \\ \operatorname{Im}\left(\xi^{(i)}\right) & r+s+1 \leqq i \leqq n\end{cases}
$$

where $\xi^{(i)}, 0 \leqq i \leqq r$, are the real conjugates of $\xi$ and if $r+1 \leqq$ $i \leqq v+s$ then $\xi^{(i)}=\bar{\xi}^{(i+s)}$. If $M$ is a module in $K$ then $\Lambda(M)=$ $\left\{\left(\xi^{*(0)}, \cdots, \xi^{*(n)}\right)\right\}$ is a lattice. Let $\left(c_{0}, \cdots, c_{n}\right) \Lambda$ be the set of all $\left(\mu_{0}, \cdots, \mu_{n}\right)$ where

$$
\mu_{i}= \begin{cases}c_{i} \lambda_{i} & 0 \leqq i \leqq r \\ c_{i} \lambda_{i}-c_{i+s} \lambda_{i+s} & r+1 \leqq i \leqq r+s \\ c_{i} \lambda_{i-s}-c_{i+s} \lambda_{i} & r+s+1 \leqq i \leqq n\end{cases}
$$

where $\left(\lambda_{0}, \cdots, \lambda_{n}\right) \in \Lambda$. If $\theta \in K$, let $\theta \Lambda=\left(\theta^{*(0)}, \cdots, \theta^{*(n)}\right) \Lambda$ so $\theta \Lambda(M)=\Lambda(\theta M)$.

We first determine $M(H)$, the number of relatively prime solutions to (6) with $H f^{-1}<\max q_{i} \leqq H$ where $f>1$ is fixed. Let $\lambda=$ $q_{1} \beta_{1}+\cdots+q_{n} \beta_{n}-p$ and let $M$ be the module consisting of all such $\lambda$, then $\Lambda(M)=\left\{\lambda^{*(0)}, \cdots, \lambda^{*(n)}\right\}$. Let $A_{1}$ be the linear transformation $A_{1}\left(p, q_{1}, \cdots, q_{n}\right)=\left(\lambda, q_{1}, \cdots, q_{n}\right)$ so $\operatorname{det}\left(A_{1}\right)=1$. Let $A_{2}=\left(a_{i j}\right), 0 \leqq$ $i, j \leqq n$, where

$$
\begin{aligned}
a_{00} & =1, \quad a_{0 j}=0, \quad a_{i 0}=0 \quad \text { and } \\
a_{i j} & = \begin{cases}\beta_{j}^{(i)}-\beta_{j} & 1 \leqq i \leqq r, \quad j \neq 0 \\
\operatorname{Re}\left(\beta_{j}^{(i)}-\beta_{j}\right) & r+1 \leqq i \leqq r+s, \quad j \neq 0 \\
\operatorname{Im}\left(\beta_{j}^{(i)}\right) & r+s+1 \leqq i \leqq n, \quad j \neq 0 .\end{cases}
\end{aligned}
$$

Let $\bar{A}_{2}=\left(a_{i j}\right), 1 \leqq i, j \leqq n$, so $\operatorname{det}\left(\bar{A}_{2}\right)=\operatorname{det}\left(A_{2}\right)= \pm \operatorname{det}(\Lambda(M))$ which is nonzero. Let

$$
\begin{aligned}
\bar{A}_{2}\left(q_{1}, \cdots, q_{n}\right)^{t} & =\left(\lambda^{*(1)}-\lambda^{*(0)}, \cdots, \lambda^{*(r+s)}-\lambda^{*(0)}, \lambda^{*(r+s+1)}, \cdots, \lambda^{*(n)}\right) \\
& =\bar{\lambda} .
\end{aligned}
$$

From (6) we see that we wish to count the number of primitive points of $\Lambda(n)$ with

$$
\begin{aligned}
& 0 \leqq \lambda^{*(0)} \leqq \varphi\left(\max \bar{A}_{2}^{-1} \bar{\lambda}\right)\left|L\left(\bar{A}_{2}^{-1} \bar{\lambda}\right)\right| \\
& \frac{H}{f}<\max \left(\bar{A}_{2}^{-1} \bar{\lambda}\right) \leqq H .
\end{aligned}
$$

We call the region of $R^{n+1}$ defined by these inequalities $D$. Its volume is given by 
(7) $\frac{V(D)}{\operatorname{det}(\Lambda(M))}=\int_{H / F<\max y_{2} \leq H ; y_{i}>0} \varphi\left(\max y_{i}\right)\left|L\left(y_{1}, \cdots, y_{n}\right)\right| d y_{1} \cdots d y_{n}$.

The lattice will be transformed as in [5].

Lemma 7. Let $M$ be a module in $K$. Then there are $\theta_{1}, \cdots, \theta_{r+s}$ in $K$ with $\theta_{j}^{(i)}>0,1 \leqq i, j \leqq r+s$ with $\operatorname{det}\left(\log \left|\theta_{j}^{(i)}\right|\right) \neq 0$ and $\theta_{j} \Lambda(M)=\Lambda(M)$.

Proof. See [5].

We let $u=\varphi(H)^{-1 /(n+1)}$. Define $\mu_{1}, \cdots, \mu_{r+s}$ by

$$
u=\left|\theta_{1}^{(i)}\right|^{\mu_{1}} \cdots\left|\theta_{r+s}^{(i)}\right|^{h_{r+s}}, \quad 1 \leqq i \leqq r+s
$$

and $m_{\imath}$ by $\left|m_{i}-\mu_{i}\right| \leqq 1 / 2$. So $\kappa=\kappa^{(0)}=\theta_{1}^{m_{1}} \cdots \theta_{r+s}^{m_{r+s}}$ is a unit of $K$ and there exists a $c$ such that

$$
\begin{aligned}
& c u \leqq \kappa^{(i)} \leqq c u, \quad 1 \leqq i \leqq n \\
& c^{-n} u^{-n} \leqq \kappa^{(0)} \leqq c^{n} u^{-n}
\end{aligned}
$$

Define $c_{\imath}$ by $\kappa^{*(0)}=u^{-n} c_{0}$ and $\kappa^{*(i)}=u c_{i}, 1 \leqq i \leqq n$. So $c_{0} \cdots c_{r}\left(c_{r+1}^{2}+\right.$ $\left.c_{r+s+1}^{2}\right) \cdots\left(c_{r+s}^{2}+c_{n}^{2}\right)=1$ and $\left|c_{i}\right| \leqq c^{n}$. Let $\Lambda^{\prime}=\left(c_{0}, \cdots, c_{n}\right) \Lambda(M)$ then $\operatorname{det} \Lambda^{\prime}=\operatorname{det} \Lambda(M)$ and $\kappa \Lambda(M)=\Lambda(M)$ is the set of all $\left(u^{-n} \nu_{0}, u \nu_{1}, \cdots\right.$, $\left.u \nu_{n}\right)$ where $\left(\nu_{0}, \cdots, \nu_{n}\right) \in \Lambda^{\prime}$. We therefore wish to count the number of primitive lattice points of $\Lambda^{\prime}$ with

$$
\left\{\begin{array}{l}
0<\nu_{0} u^{-n}<\left(\max \bar{A}_{2}^{-1} \bar{\lambda}\right) L\left(\bar{A}_{2}^{-1} \bar{\lambda}\right) \\
H f^{-1}<\max \bar{A}^{-1} \bar{\lambda} \leqq H
\end{array}\right.
$$

where $\bar{\lambda}=\left(u \nu_{1}-u^{-n} \nu_{0}, \cdots, u \nu_{n}-u^{-n} \nu_{0}\right)$. Let $\bar{A}_{2}^{-1} \bar{\lambda}=u \bar{\gamma}-u^{-n} \nu_{0} \bar{\gamma}_{0}$ where $\bar{\gamma}, \bar{\gamma}_{0} \in \boldsymbol{R}^{n}$. Since $u^{-n} \nu_{0}$ is bounded, if we count the number of solutions to

$$
\left\{\begin{array}{l}
0<\nu_{0} u^{-n}<\varphi(u \bar{\gamma})|L(u \bar{\gamma})| \\
H f^{-1}<\max (u \bar{\gamma}) \leqq H
\end{array}\right.
$$

instead of (8) we will get an error term which is a constant times the volume of the boundary of the region given by (9), which can be absorbed into the error term we will get. The actual counting is done in the next lemma.

Lemma 8. Let $\Lambda$ be a lattice, $D$ a bounded set in $\boldsymbol{R}^{n+1}$, $\varepsilon$ the minimum diameter ef all fundamental parallelepipeds of $\Lambda$ and $D(\varepsilon)$ the set of all points with distance at most $\varepsilon$ from the boundary of $D$. If $n \geqq 2$ the number of primitive lattice points of $D$ is given by 


$$
\left(\sum_{k=1}^{\infty} \frac{\mu(k)}{k^{n+1}}\right) \frac{\operatorname{Vol}(D)}{\operatorname{det} \Lambda}+O(\operatorname{Vol}(D(\varepsilon))) .
$$

Proof. The proof follows easily from Lemma 4 in [5].

We now let $\pi(y)=y \varphi(y)^{1 / n+1}$ and from (9) we can check that $\operatorname{Vol}(D(\varepsilon))=O\left(\pi(H)^{n}\right)$ and

$$
\pi(H)^{n}=O\left(\int_{H / f^{2}}^{H / f} y^{n} \varphi(y) \chi\left(f^{2} y\right)^{-1 /(n+1)} d y\right) .
$$

If $H_{0}>f^{2}$ is fixed and if $H_{0} f<B$ we get

$$
N(B)=M(B)+M(B / f)+M\left(B / f^{2}\right)+\cdots+M\left(H_{0}\right)+O(1) .
$$

From Lemma 8 and (7) we then get

$$
\begin{aligned}
N(B)= & C \int_{1 \leqq y_{i} \leqq B} \varphi\left(\max y_{i}\right)\left|L\left(y_{1}, \cdots, y_{n}\right)\right| d y_{1}, \cdots, d y_{n} \\
& +O\left(\int_{1}^{B / f} y^{n} \varphi(y) \chi\left(f^{2} y\right)^{-1 /(n+1)} d y\right)
\end{aligned}
$$

with $C=\sum_{k=1}^{\infty} \mu(k) / k^{n+1}$ and this proves Theorem $1^{\prime}$.

To prove Theorem 1 we must count solutions for each possible choice of sign for $\left(p, q_{1}, \cdots, q_{n}\right)$ which can be done by letting $\beta_{i}=$ $\pm \beta^{i}$. We must also check that the error terms from Theorems $1^{\prime}$ and 3 give the desired error.

4. The proof of Theorem 2, Instead of Theorem 2 we prove the following from which Theorem 2 follows.

TheORem 2 . Let $1, \beta_{1}, \cdots, \beta_{n}$ be as in $\S 3$. The number of solutions to

$$
0<q_{1} \beta_{1}+\cdots+q_{n} \beta_{n}-p<\left(\sum_{\imath=1}^{n} c_{i} q_{i}\right)\left(\max q_{\imath}\right)^{-(n+1)}, \quad 1 \leqq q_{i} \leqq B
$$

where $c_{i} \in \boldsymbol{R}$, is either $O(1)$ or asymptotic to $C \log B$, for some $C>0$.

The proof of the theorem is similar to those of Adams in $[1,2]$ and so it will only be sketched. Let $\lambda=q_{1} \beta_{1}+q_{2} \beta_{2}+\cdots+q_{n} \beta_{n}-p$, $K, M$ be as before. Let $\theta$ be the associated order, i.e., $\theta=\{\alpha \in K$ : $\alpha M \subset M\}$. By Dirichlet's Unit Theorem there exists units $\zeta_{1}, \cdots$, $\zeta_{r+s}>1$ such that the unit group is $U=\left\{ \pm \zeta_{1}^{\nu_{1}} \zeta_{2}^{\nu} \cdots \zeta_{r+1}^{\nu}\right\}=\left\{ \pm \zeta_{0}^{\nu}\right\}$ where $\nu=\left(\nu_{1}, \cdots, \nu_{r_{s}}\right)$. We say $\lambda_{1}$ is equivalent to $\lambda_{2}$ if $\lambda_{1}=\zeta \lambda_{2}$ for some $\zeta \in U$. This is an equivalence relation. We note that the $\lambda \in M$ correspond to the possible solutions of (10). As in [2] it is easy to see that we need only count the number of solutions in a fixed equivalence class and we let $\lambda_{\nu}=\zeta_{0}^{\nu} \lambda_{0}$ with $\lambda_{0}>0$, since by (10) we must 
have $\lambda_{\nu}>0$. Write

$$
\lambda_{\nu}=q_{1 \nu} \beta_{1}+\cdots+q_{n \nu} \beta_{n}-p_{\nu}
$$

and let $\delta_{i \nu}=q_{i \nu} \lambda_{\nu}^{1 / n}$. If $\max q_{i \nu}$ is large we can assume $q_{i \nu} \neq 0$, for all $i$, since otherwise we would contradict Schmidt's theorem [6]. We have (10) is equivalent to

$$
0<\delta_{\imath \nu}^{n}<\min _{j}\left(\sum_{k=1}^{n} c_{k} \delta_{k \nu} \delta_{j \nu}^{-1}\right), \quad 1 \leqq q_{i \nu} \leqq B .
$$

Let $R$ be the region defined by (11), then one can check that $R$ is bounded. Let $A_{3}$ be the matrix $\left(\beta_{j}^{(i)}-\beta_{j}\right), 1 \leqq i, j \leqq n . \quad A_{3}$ is nonsingular since $\left(\operatorname{det} A_{3}\right)^{2}$ is the discriminant of $1, \beta_{1}, \cdots, \beta_{n}$. Define $A_{4}=\left(a_{i j}\right)$ by

$$
a_{\imath j}= \begin{cases}0 & \text { if } \quad i \neq j \\ \lambda_{0}^{-1 / n} \lambda_{0}^{(2)-1} & \text { if } \quad i=j\end{cases}
$$

Then

$$
\begin{aligned}
P_{\nu} & =A_{4} A_{3}\left(\delta_{1 \nu}, \cdots, \delta_{n \nu}\right) \\
& =\left(\zeta^{\nu / n} \zeta^{(1) \nu}-\lambda_{\nu} \lambda_{0}^{(1)^{-1}}, \cdots, \zeta^{\nu / n} \zeta^{(n)^{\nu}}-\lambda_{\nu} \lambda_{0}^{(n)^{-1}}\right)
\end{aligned}
$$

is near

$$
Q_{\nu}=\left(\zeta^{\nu / n} \zeta^{(1)^{\nu}}, \cdots, \zeta^{\nu / n} \zeta^{(n)^{\nu}}\right) .
$$

Since the last $s$ coordinates of $Q$, are the complex conjugates of the preceding $s$ coordinates we can omit them to get a nonsingular transformation

$$
\boldsymbol{R}^{n} \longrightarrow \boldsymbol{R}^{r} \times \boldsymbol{C}^{2 s} \longrightarrow \boldsymbol{R}^{r} \times \boldsymbol{C}^{s} \longrightarrow \boldsymbol{R}^{n} .
$$

We can now apply the uniform distribution theory to count the number of $Q_{\nu}$ in the region $R^{\prime}=A_{4} A_{3} R$. This is done exactly as in Lemma 4 of [2] and so we omit it. The result is:

Lemma 9. The number of $Q_{\nu}$ in $R^{\prime}$ with $1 \leqq \nu_{1} \leqq N$ is asymptotic to $C N$ or $O(1)$ and the same is true for $1 \leqq-\nu_{1} \leqq N$.

To show it is alright to count the $Q_{2}$ instead of the $P_{\nu}$, we prove two lemmas.

Lemma 10. If $P_{\nu} \in R^{\prime}$ then $\max q_{i \nu} \doteq \max \left|\lambda_{\nu}^{(2)}\right|$ where $A \doteq B$ means $A \ll B$ and $B \ll A$.

Proof. Since $\lambda$, is small

$$
A_{4} A_{3}\left(q_{1 \nu}, \cdots, q_{n \nu}\right)=\left(\lambda_{\nu}^{(1)}-\lambda_{\nu}, \cdots, \lambda_{\nu}^{(n)}-\lambda_{\nu}\right)
$$


implies $\max q_{i \nu} \ll \max \left|\lambda_{\nu}^{(i)}\right|$. Since $A_{4} A_{3}$ is invertible we also get the reverse inequality.

LEMMa 11. If $P_{\nu}$ or $Q_{\nu}$ are in $R^{\prime}$ then

$$
\log \max \left|\lambda_{\nu}^{(i)}\right|=C_{1} \nu+O(1)
$$

where $C_{1} \neq 0$.

Proof. If we let $X_{i}=\left(\log \left|\zeta_{i}^{1 / n} \zeta_{i}^{(2)}\right|, \cdots, \log \left|\zeta_{i}^{1 / n} \zeta_{i}^{(r+s)}\right|\right)$ then as in Lemma 5 of [2] we get

$$
\begin{aligned}
\log \left|\lambda_{\nu}^{(i)}\right|= & \nu_{1} \frac{\left(\sum_{j=1}^{r+s}(-1)^{j-1} \operatorname{det}\left(X_{1}, \cdots, \hat{X}_{j}, \cdots, X_{r+s}\right)\left(\log \left|\zeta_{j}^{(i)}\right|\right)\right)}{\operatorname{det}\left(X_{2}, \cdots, X_{r+s}\right)} \\
& +O(1) .
\end{aligned}
$$

The denominator is nonzere as in [2] and, for any choice of $i$, the numerator is $1 / n$ times the regulator and so nonzero and independent of $i$, which proves the lemma.

Thus we have that as $\left|\nu_{1}\right| \rightarrow \infty$ we must have $\max \left|\lambda_{\nu}^{(i)}\right| \rightarrow \infty$ and so $\lambda_{\nu} \rightarrow 0$ which implies $\left|P_{\nu}-Q_{\nu}\right| \rightarrow 0$.

The proof is now finished as in [2]. The number of $P$ in $R^{\prime}$ with $1 \leqq\left|\nu_{1}\right| \leqq N$ is shown to be asymptotic to $C N$. Letting $N=\left|C_{1}\right|^{-1} \log B$ this gives $1 \leqq q_{i \nu} \leqq B$ is equivalent to $O(1) \leqq\left|C_{1} \nu_{1}\right| \leqq \log B+O(1)$. The number of solutions to (10) is then asymptotically the same if $1 \leqq q_{i \nu} \leqq B$ is replaced by $1 \leqq\left|\nu_{1}\right| \leqq\left|C_{1}\right|^{-1} \log B$ and this is $C N$ or $C^{\prime} \log B$ as desired.

To prove Theorem 2 we must show that adding the restriction that the coefficients of $P$ be relatively prime does not change the form of the result. To do this we note that there is a $C_{0}$ such that $0<P(\beta)<C_{0}\left|P^{\prime}(\beta)\right| H(P)^{-(n+1)}$ has only a finite number of solutions. Thus if $d$ divides all the coefficients of $P$ and $0<P(\beta)<$ $C\left|P^{\prime}(\beta)\right| H(P)^{-(n+1)}$ then $d^{n+1}<C_{0}^{-1} C$, and so the set of all such $d$ is finite. Let $d_{0}$ be the largest such integer, then it is not hard to show that if the number of soultions to $0<P(\beta)<C d^{-(n+1)}\left|P^{\prime}(\beta)\right| H(P)^{-(n+1)}$ is asymptotic to $C_{d} \log B$ then the number of primitive solutions with $d=1$ is asymptotic to $\left(\sum_{d=1}^{d_{0}} \mu(d) C_{d}\right) \log B$. Theorems $2^{\prime}$ and 3 can then be combined to give Theorem 2 .

\section{REFERENCES}

1. W. W. Adams, Simultaneous asymptotic diophantine approximations to a basis of a real cubic number field, J. Number Theory, 1 (1969), 179-194.

2. Simultaneous asymptotic diophantine approximations to a basis of a real number field, Nagoya Math. J., 42 (1971), 79-87.

3. A. O. Gelfand, Transcendental and Algebraic Numbers, Dover, New York, 1960. 
4. J. F. Morrison, Approximation of p-adic numbers by algebraic numbers of bounded degree, J. Number Theory, 10 (1978), 334-350.

5. W. M. Schmidt, Simultaneous approximation to a basis of a real numberfield, Amer. J. Math., 88 (1966), 517-527.

6 . Simultaneous approximation to algebraic numbers by rationals, Acta Math., 125 (1970), 189-201.

Received November 9, 1979. Publication costs were provided by the Faculty Research Committee of Towson State University.

Towson State University

Baltimore, MD 21204 



\section{PACIFIC JOURNAL OF MATHEMATICS}

\section{EDITORS}

DONALD BABBITT (Managing Editor)

University of California

Los Angeles, CA 90024

HUGo RossI

University of Utah

Salt Lake City, UT 84112

C. C. MOORE and ANDREW OGG

University of California

Berkeley, CA 94720
J. DUGUNDJI

Department of Mathematics

University of Southern California

Los Angeles, CA 90007

R. FinN and J. Milgram

Stanford University

Stanford, CA 94305

\section{ASSOCIATE EDITORS}
R. ARENS
E. F. BECKENBACH
B. H. NeumanN
F. WOLF
K. Yoshida

\section{SUPPORTING INSTITUTIONS}

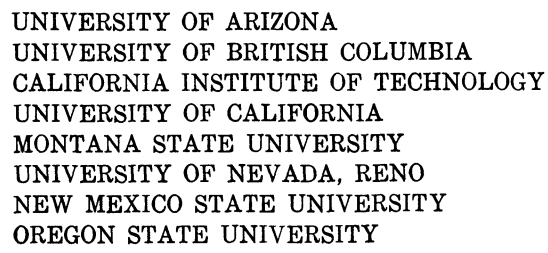

UNIVERSITY OF ARIZONA

UNIVERSITY OF BRITISH COLUMBIA CALIFORNIA INSTITUTE OF TECHNOLOGY

UNIVERSITY OF CALIFORNIA

MONTANA STATE UNIVERSITY

UNIVERSITY OF NEVADA, RENO

NEW MEXICO STATE UNIVERSITY

OREGON STATE UNIVERSITY

\author{
UNIVERSITY OF OREGON \\ UNIVERSITY OF SOUTHERN CALIFORNIA \\ STANFORD UNIVERSITY \\ UNIVERSITY OF HAWAII \\ UNIVERSITY OF TOKYO \\ UNIVERSITY OF UTAH \\ WASHINGTON STATE UNIVERSITY \\ UNIVERSITY OF WASHINGTON
}

The Supporting Institutions listed above contribute to the cost of publication of this Journal, but they are not owners or publishers and have no responsibility for its content or policies.

Mathematical papers intended for publication in the Pacific Journal of Mathematics should be in typed form or offset-reproduced, (not dittoed), double spaced with large margins. Please do not use built up fractions in the text of the manuscript. However, you may use them in the displayed equations. Underline Greek letters in red, German in green, and script in blue. The first paragraph or two must be capable of being used separately as a synopsis of the entire paper. Please propose a heading for the odd numbered pages of less than 35 characters. Manuscripts, in triplicate, may be sent to any one of the editors. Please classify according to the scheme of Math. Reviews, Index to Vol. 39. Supply name and address of author to whom proofs should be sent. All other communications should be addressed to the managing editor, or Elaine Barth, University of California, Los Angeles, California, 90024.

50 reprints to each author are provided free for each article, only if page charges have been substantially paid. Additional copies may be obtained at cost in multiples of 50 .

The Pacific Journal of Mathematics is issued monthly as of January 1966. Regular subscription rate: $\$ 102.00$ a year (6 Vols., 12 issues). Special rate: $\$ 51.00$ a year to individual members of supporting institutions.

Subscriptions, orders for numbers issued in the last three calendar years, and changes of address shoud be sent to Pacific Journal of Mathematics, P.O. Box 969, Carmel Valley, CA 93924, U.S.A Old back numbers obtainable from Kraus Periodicals Co., Route 100, Millwood, NY 10546.

\section{PUBLISHED BY PACIFIC JOURNAL OF MATHEMATICS, A NON-PROFIT CORPORATION}

Printed at Kokusai Bunken Insatsusha (International Academic Printing Co., Ltd.). 8-8, 3-chome, Takadanobaba, Shinjuku-ku, Tokyo 160, Japan. 


\section{Pacific Journal of Mathematics}

Vol. 92, No. $2 \quad$ February, 1981

Bruce Allem Anderson and Philip A. Leonard, Sequencings and Howell designs

Kevin T. Andrews, Representation of compact and weakly compact

operators on the space of Bochner integrable functions . . . . . . . . 257

James Glenn Brookshear, On the structure of hyper-real $z$-ultrafilters . . . . . 269

Frank John Forelli, Jr., A necessary condition on the extreme points of a class of holomorphic functions. II ...................... 277

Richard J. Friedlander, Basil Gordon and Peter Tannenbaum, Partitions of groups and complete mappings ......................... 283

Emden Robert Gansner, Matrix correspondences of plane partitions ......295

David Andrew Gay and William Yslas Vélez, The torsion group of a radical extension ..........................................

André (Piotrowsky) De Korvin and C. E. Roberts, Convergence theorems for some scalar valued integrals when the measure is Nemytskii ...... 329

Takaŝi Kusano and Manabu Naito, Oscillation criteria for general linear ordinary differential equations $\ldots \ldots \ldots \ldots \ldots \ldots \ldots \ldots \ldots \ldots \ldots \ldots \ldots \ldots \ldots \ldots$

Vo Thanh Liem, Homotopy dimension of some orbit spaces .......... 357

Mark Mahowald, $b o$-resolutions . . . . . . . . . . . . . . . . . . . 365

Jan van Mill and Marcel Lodewijk Johanna van de Vel, Subbases, convex

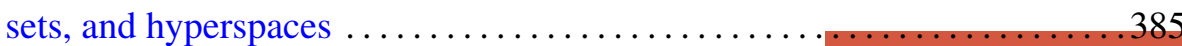

John F. Morrison, Approximations to real algebraic numbers by algebraic numbers of smaller degree $\ldots \ldots \ldots \ldots \ldots \ldots \ldots \ldots \ldots \ldots \ldots \ldots \ldots$

Caroline Series, An application of groupoid cohomology . . . . . . . . . . 415

Peter Frederick Stiller, Monodromy and invariants of elliptic surfaces . . . 433 Akihito Uchiyama, The factorization of $H^{p}$ on the space of homogeneous

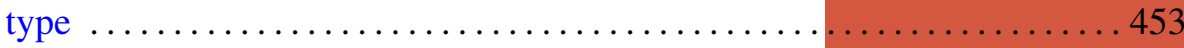

Warren James Wong, Maps on simple algebras preserving zero products.

II. Lie algebras of linear type 